\title{
Correction to: Characterising cognitive heterogeneity in individuals at clinical high-risk for psychosis: a cluster analysis with clinical and functional outcome prediction
}

\author{
Kate Haining ${ }^{1} \cdot$ Ruchika Gajwani ${ }^{2}$. Joachim Gross ${ }^{1,3}$. Andrew I. Gumley ${ }^{2} \cdot$ Robin A. A. Ince $^{1} \cdot$ Stephen M. Lawrie ${ }^{4}$. \\ Frauke Schultze-Lutter ${ }^{5,6,7} \cdot$ Matthias Schwannauer $^{8} \cdot$ Peter J. Uhlhaas $^{1,9}$
}

Published online: 14 September 2021

(c) Springer-Verlag GmbH Germany, part of Springer Nature 2021

\section{Correction to: \\ European Archives of Psychiatry and Clinical Neuroscience \\ https://doi.org/10.1007/s00406-021-01315-2}

In the original article published, during typesetting, an erroneous correction in table 2 was performed. The word "Follow-up" has been changed to "Baseline". Please see the correction of Table 2 below."

The original article has been corrected.

The original article can be found online at https://doi.org/10.1007/ s00406-021-01315-2.

Peter J. Uhlhaas

peter.uhlhaas@charite.de

1 Institute of Neuroscience and Psychology, University

of Glasgow, Glasgow, UK

2 Institute of Health and Wellbeing, University of Glasgow, Glasgow, UK

3 Institute for Biomagnetism and Biosignalanalysis, University of Münster, Münster, Germany

4 Department of Psychiatry, University of Edinburgh, Edinburgh, UK

5 Department of Psychiatry and Psychotherapy, Medical Faculty, Heinrich Heine University, Düsseldorf, Germany

6 Department of Psychology and Mental Health, Faculty of Psychology, Airlangga University, Surabaya, Indonesia

7 University Hospital of Child and Adolescent Psychiatry and Psychotherapy, University of Bern, Bern, Switzerland

8 Department of Clinical Psychology, University of Edinburgh, Edinburgh, UK

9 Department of Child and Adolescent Psychiatry, Charité Universitätsmedizin, Berlin, Germany 
Table 2 Demographic, clinical and functional characteristics of the CHR-P group by cognitive cluster at baseline $(N=146)$ and follow-up $(N=122)$

\begin{tabular}{|c|c|c|c|c|}
\hline Baseline & $\begin{array}{l}\text { Cluster } 1 \\
\text { Impaired }(N=67)\end{array}$ & $\begin{array}{l}\text { Cluster } 2 \\
\text { Spared }(N=79)\end{array}$ & $p$ & Effect size $^{\mathrm{a}}$ \\
\hline Age (years), mean (SD) & $21.36(4.63)$ & $21.56(3.86)$ & 0.288 & $r=0.088$ \\
\hline Sex, female $n(\%)$ & $35(52.2)$ & $69(87.3)$ & $<0.001$ & $\varphi=0.386$ \\
\hline Education (years), mean (SD) & $14.96(3.43)$ & $15.25(2.80)$ & 0.421 & $r=0.067$ \\
\hline CAARMS severity, median (range) & $29(0-74)$ & $28(0-72)$ & 0.212 & $r=0.103$ \\
\hline SPI-A severity, median (range) & $6(0-61)$ & $7(0-74)$ & 0.883 & $r=0.012$ \\
\hline GAF, median (range) & $55(21-87)$ & $60(21-95)$ & 0.094 & $r=0.139$ \\
\hline Social functioning (current), median (range) & $7(3-10)$ & $8(3-10)$ & $<0.001$ & $r=0.296$ \\
\hline Role functioning (current), median (range) & $7(4-9)$ & $8(3-9)$ & 0.002 & $r=0.255$ \\
\hline PAS average, median (range) & $1.36(0-3.43)$ & $0.86(0-2.57)$ & $<0.001$ & $r=0.405$ \\
\hline \multicolumn{5}{|l|}{ Comorbidity, $n(\%)$} \\
\hline Anxiety disorder & $49(73.1)$ & $55(69.6)$ & 0.640 & $\varphi=0.039$ \\
\hline Mood disorder & $50(74.6)$ & $47(59.5)$ & 0.054 & $\varphi=0.160$ \\
\hline Alcohol abuse/dependence & $18(26.9)$ & $28(35.4)$ & 0.266 & $\varphi=0.092$ \\
\hline Substance abuse/dependence & $11(16.4)$ & $13(16.5)$ & 0.995 & $\varphi=0.001$ \\
\hline Eating disorder & $4(6.0)$ & $9(11.4)$ & 0.252 & $\varphi=0.095$ \\
\hline \multicolumn{5}{|l|}{ Psychological treatment, $n(\%)$} \\
\hline Current & $15(22.4)$ & $10(12.7)$ & 0.120 & $\varphi=0.129$ \\
\hline Past & $27(40.3)$ & $39(49.4)$ & 0.273 & $\varphi=0.091$ \\
\hline \multicolumn{5}{|l|}{ Medication, $n(\%)$} \\
\hline Antidepressants & $25(37.3)$ & $28(35.4)$ & 0.815 & $\varphi=0.019$ \\
\hline Mood stabilisers & $2(3.0)$ & $2(2.5)$ & 1.000 & $\varphi=0.014$ \\
\hline Antipsychotics & $3(4.5)$ & $1(1.3)$ & 0.333 & $\varphi=0.098$ \\
\hline Anxiolytics & $4(6.0)$ & $6(7.6)$ & 0.754 & $\varphi=0.032$ \\
\hline \multirow[t]{2}{*}{ Follow-up } & Cluster 1 & Cluster 2 & $p$ & Effect size $^{\mathrm{a}}$ \\
\hline & Impaired $(N=57)$ & Spared $(N=65)$ & & \\
\hline GAF, median (range) & $52(21-88)$ & $68(33-88)$ & 0.012 & $r=0.227$ \\
\hline Poor functional outcome, $n(\%)$ & $41(71.9)$ & $31(47.7)$ & 0.007 & $\varphi=0.246$ \\
\hline Social functioning (current), median (range) & $8(2-10)$ & $8(4-9)$ & 0.021 & $r=0.209$ \\
\hline Role functioning (current), median (range) & $8(4-9)$ & $8(5-9)$ & 0.139 & $r=0.134$ \\
\hline CAARMS severity, median (range) & $15(0-71)$ & $12(0-82)$ & 0.886 & $r=0.013$ \\
\hline CAARMS persistence, $n(\%)$ & $17(29.8)$ & $21(32.3)$ & 0.768 & $\varphi=0.027$ \\
\hline Transitions $^{\mathrm{b}}, n(\%)$ & $9(15.8)$ & $5(7.7)$ & 0.162 & $\varphi=0.127$ \\
\hline
\end{tabular}

CHR-P clinical high-risk for psychosis; $C A A R M S$ Comprehensive Assessment of At-Risk Mental States; SPI-A Schizophrenia Proneness Instrument, Adult version; GAF Global Assessment of Functioning; PAS premorbid adjustment scale

${ }^{a}$ Effect sizes were Rosenthal's $r$ for Mann-Whitney $U$ tests and Phi $(\phi)$ for Pearson's chi-square or Fisher's exact tests (small effect $=0.1$, medium effect $=0.3$, large effect $=0.5$ )

${ }^{\mathrm{b}} 19$ non-transitioned CHR-P individuals have yet to reach the 3-year follow-up 